\title{
Simple Qualitative Sensory Assessment of Patients with Orofacial Sensory Dysfunction
}

\author{
Yeong-Gwan Im, Byung-Gook Kim, Jae-Hyung Kim \\ Department of Oral Medicine, Dental Science Research Institute, School of Dentistry, \\ Chonnam National University, Gwangju, Korea
}

Received December 8, 2021

Revised December 17, 2021

Accepted December 18, 2021
Correspondence to:

Jae-Hyung Kim

Department of Oral Medicine, Dental Science Research Institute, School of Dentistry,

Chonnam National University, 33 Yongbong-

ro, Buk-gu, Gwangju 61186, Korea

Tel: +82-62-530-5844

Fax: $+82-62-530-5679$

E-mail: tmjkim@jnu.ac.kr

https://orcid.org/0000-0001-5646-4371
Purpose: Oral and facial sensation is affected by various factors, including trauma and disease. This study assessed the clinical profile of patients diagnosed with sensory dysfunction and investigated their sensory perception using simple qualitative sensory tests.

Methods: Based on a retrospective review of the medical records, we analyzed a total of 68 trigeminal nerve branches associated with sensory dysfunction in 52 subjects. We analyzed the frequency and etiology of sensory dysfunction, and the frequency of different types of sensory perception in response to qualitative sensory testing using tactile and pin-prick stimuli.

Results: The inferior alveolar nerve branch was the most frequently involved in sensory dysfunction (88.5\%). Third molar extraction (36.5\%) and implant surgery (36.5\%) were the most frequent etiological factors associated with sensory dysfunction. Hypoesthesia was the most frequent sensory response to tactile stimuli (60.3\%). Pin-prick stimuli elicited hyperalgesia, hypoalgesia, and analgesia in 32.4\%, 27.9\%, and 36.8\%, respectively. A significant association was found between the two kinds of stimuli $(p=0.260)$.

Conclusions: Sensory dysfunction frequently occurs in the branches of the trigeminal nerve, including the inferior alveolar nerve, mainly due to trauma associated with dental treatment. Simple qualitative sensory testing can be conveniently used to screen sensory dysfunction in patients with altered sensation involving oral and facial regions.

Key Words: Sensation; Sensory testing; Trigeminal nerve; Trigeminal nerve injuries

\section{INTRODUCTION}

Oral and facial nerves are susceptible to damage by various causes. The etiology of peripheral nerve damage in the oral and facial areas includes facial fractures, infections, tumors, and non-iatrogenic causes such as systemic diseases and genetic factors, and iatrogenic injuries, such as extraction of the third molar, implant surgery, periodontal surgery, and orthognathic surgery [1-7]. Damage to the trigeminal nerve elicits neurologic signs and symptoms associated with sensory dysfunction in the oral and facial areas
$[3,8]$.

Evaluation of somatosensory function is recommended as part of a comprehensive examination in patients with orofacial sensory impairment. The mapping procedure is intended to limit the area of somatosensory changes and to identify the affected nerves. Marking the boundary between "normal" and "conditional" sensations based on the subject's response reveals areas of sensory change [9]. Quantitative sensory testing (QST) is a valuable tool in psychophysical examination for evaluation of the neurosensory function in the oral and facial region $[10,11]$. A

Copyright (C) 2021 Korean Academy of Orofacial Pain and Oral Medicine.

(c) This is an open-access article distributed under the terms of the Creative Commons Attribution Non-Commercial License (http://creativecommons.org/licenses/by-nc/4.0/), which permits unrestricted non-commercial use, distribution, and reproduction in any medium, provided the original work is properly cited. 
comprehensive QST battery includes tests for thermal and mechanical detection of pain threshold, evaluation of stimulus-response function, temporal summation of pain, and determination of threshold of vibration detection and pressure pain detection $[12,13]$. Clinical neurophysiological testing and QST are recommended for the diagnosis of trigeminal neuropathy due to their diagnostic accuracy [14]. QST is also useful for evaluating the recovery of sensory nerve function over time [15]. However, these tests are time-consuming and require specialized instruments and equipment.

Although not as sensitive as QST, qualitative sensory testing (QualST) based on touch, cold, and pin-prick can be performed quickly and inexpensively in primary care settings. Tactile stimulation is used to evaluate the function of myelinated $A \beta$ fibers, while pin-pricks are used to assess thin myelinated $A \delta$ and unmyelinated $C$ fibers [16]. Simple QualST was found to be reliable for the purpose of initial screening to evaluate the orofacial somatosensory function [17].

Therefore, in this study, we investigated the clinical profile of patients with orofacial sensory dysfunction and evaluated the outcomes of simple QualST to characterize sensory perception.

\section{MATERIALS AND METHODS}

This study was conducted using a retrospective chart review, and therefore exempted from ethics approval by the Chonnam National University Dental Hospital Institutional Review Board.

Patients presenting with complaints of sensory disturbance in the oral and facial regions at the Department of Oral Medicine, Chonnam National University Dental Hospital from January 2014 to January 2021 were included as study subjects. Included subjects were 19 years or older who had complete medical records and results of QualST. Subjects with known systemic diseases or central nervous system pathologies that could result in somatosensory impairment of the body were excluded. Patients were also excluded if they showed normal findings on QualST. A total of 52 subjects (27 females and 25 males; mean age $54.0 \pm 16.0$ years) met the inclusion criteria and thus represented the study group.

The patients' history including the location and quality of symptoms, the causative events or agents, and the elapsed time since the onset of symptoms was reviewed. The clinical findings were: (1) sensory dysfunction associated with the trigeminal nerve and (2) the results of QualST using two types of stimuli administered to the area of sensory dysfunction.
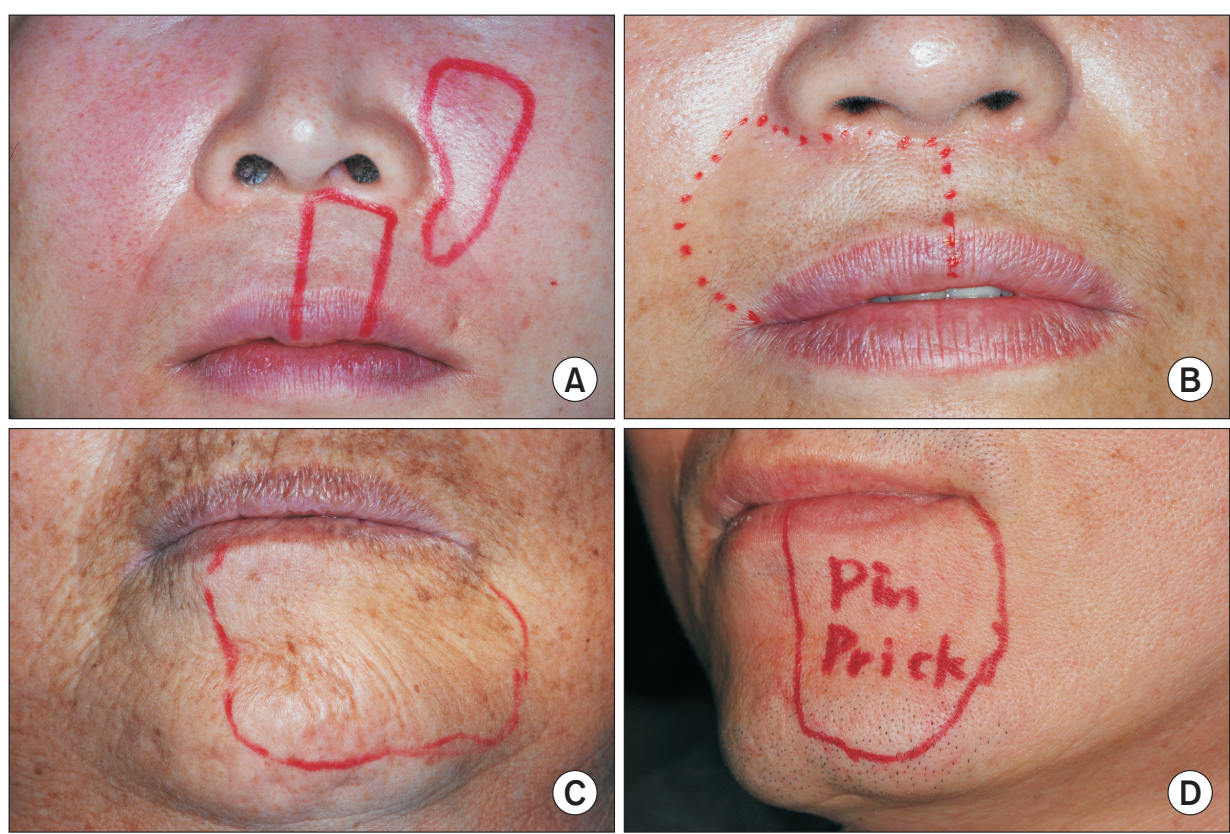

Fig. 1. Visual mapping of the areas with sensory dysfunction of perioral and facial skin and identification of the affected nerve branches. (A) Infraorbital nerve region affected by maxillofacial infection. (B) Infraorbital nerve region affected by periodontal surgery. (C) Inferior alveolar nerve region affected by odontogenic infection. (D) Inferior alveolar nerve region affected by the extraction of a third molar. 
The affected nerve was identified based on the results of QualST and possible visual mapping of the areas of sensory dysfunction in the facial skin. Clinical examination including QualST and mapping of sensory dysfunction was performed with subjects in the supine position, with headrest and backrest of the dental chair flattened to the floor. Subjects were asked to report the sensation to a light touch with a cotton swab on the test sites. The border between normal and sensory dysfunction was demarcated on the skin using a cosmetic pencil. Specific nerve branches were matched to the demarcated area according to the known innervation pattern of the trigeminal nerve (Fig. 1). In 16 of 52 subjects, there was sensory dysfunction involving two independent branches of the trigeminal nerve ipsilaterally. Therefore, a total of 68 nerve branches were included in the analysis of the QualST results.

QualST was used to assess subjects' sensory perception to tactile and pin-prick stimuli. For tactile stimulation, a cotton swab was used to provide a light touch briefly to the superficial skin of test sites without pressing down. For pinprick stimulation, a stainless-steel dental explorer with a curved hook was used. The pointed tip of the dental explorer was dropped onto test sites from 2 to $3 \mathrm{~cm}$ above without piercing the skin. The stimulation was alternatively applied within the marked area and outside the area on the opposite side. Subjects' reported sensory perception to tactile stimuli was interpreted as normal, hyperesthesia, hypoesthesia, and anesthesia. Abnormal tingling sensation to tactile stimuli was considered hyperesthesia. Subjects' responses to pinprick stimuli were interpreted as normal, hyperalgesia, hypoalgesia, and analgesia (Table 1).

\section{Statistical Analysis}

The frequency of the involved nerve branches, etiologic factors underlying sensory dysfunction, and the elapsed time from the onset of symptoms were analyzed. The results of QualST evaluating the frequency of sensory response to tactile and pin-prick stimuli were analyzed. Fisher's exact test was used to assess the association between the nerve branches involved and QualST results. Furthermore, the association between sensory response to tactile and pin-prick stimuli was analyzed. All analyses were statistically analyzed using SPSS, version 21.0 (IBM Corp., Armonk, NY, USA), and the difference was regarded as significant at a pvalue less than 0.05 .

\section{RESULTS}

The identified nerve branches with sensory dysfunction of the 52 subjects included the infraorbital branch of the maxillary nerve, and the buccal, inferior alveolar, and lingual branches of the mandibular division of the trigeminal nerve. Among them, 16 had sensory dysfunction involving two independent branches of the trigeminal nerve on the same side.

The inferior alveolar nerve branch (46 subjects, 88.5\%) was the most frequently involved in sensory dysfunction. The mandibular nerve branches were involved in 84.6\% of the subjects and the maxillary nerve in 15.4\% (Table 2). Sensory dysfunction was identified in 32 subjects (61.5\%) on the left side and 20 (38.5\%) on the right side.

Most subjects manifested traumatic injury. Six subjects (11.5\%) had sensory dysfunction resulting from local infection of odontogenic or non-odontogenic origin. The most frequent etiologic factors were third molar extraction (36.5\%) and implant surgery (36.5\%). Factors related to dental treatment, such as extraction of third molars, implant surgery, endodontic surgery, periodontal surgery, and orthognathic surgery accounted for $88.5 \%$. Sensory dysfunction occurred as a complication of surgical treatment in

Table 1. Definition of terms related to perception of sensory stimuli

\begin{tabular}{|c|c|c|}
\hline Sensory stimuli & Term & Definition \\
\hline \multirow[t]{3}{*}{ Tactile stimulus } & Hyperesthesia & Increased sensitivity to stimulation \\
\hline & Hypoesthesia & Decreased sensitivity to stimulation \\
\hline & Anesthesia & Absence of sensation to stimulation \\
\hline \multirow[t]{3}{*}{ Pin-prick stimulus } & Hyperalgesia & Increased sensitivity to noxious stimulation \\
\hline & Hypoalgesia & Diminished sensitivity to noxious stimulation \\
\hline & Analgesia & Absence of pain in response to noxious stimulation \\
\hline
\end{tabular}


Table 2. Etiologic factors associated with sensory dysfunction of involved branches of the trigeminal nerve

\begin{tabular}{|c|c|c|c|c|c|}
\hline \multirow{2}{*}{ Factor } & \multirow{2}{*}{$\frac{\text { Maxillary nerve }}{\text { Infraorbital branch }}$} & \multicolumn{3}{|c|}{ Mandibular nerve } & \multirow{2}{*}{ Total } \\
\hline & & Buccal branch & Inferior alveolar branch & Lingual branch & \\
\hline Infection & 2 & 1 & 3 & & $6(11.5)$ \\
\hline Physical trauma & 2 & 1 & 7 & 1 & $11(21.2)$ \\
\hline Endodontic surgery & 2 & & & & $2(3.8)$ \\
\hline Periodontal surgery & 1 & & 3 & & $4(7.7)$ \\
\hline Third molar extraction & & 1 & 10 & 8 & $19(36.5)$ \\
\hline Implant surgery & 1 & & 17 & 1 & $19(36.5)$ \\
\hline Orthognathic surgery & & & 2 & & $2(3.8)$ \\
\hline Surgical treatment of a cystic lesion & & & 3 & 1 & $4(7.7)$ \\
\hline Surgical treatment of a cancer lesion & & & 1 & & $1(1.9)$ \\
\hline Total & $8(15.4)$ & $3(5.8)$ & $46(88.5)$ & $11(21.2)$ & 68 \\
\hline
\end{tabular}

Values are presented as number (\%). Number of the subjects=52 (females, 27; males, 25; mean age 54.0 \pm 16.0 ). Sensory dysfunction involving two independent branches of the trigeminal nerve on the same side was reported in 16 of 52 subjects. The ratio was calculated as the number of affected nerve branches relative to the total number of patients.

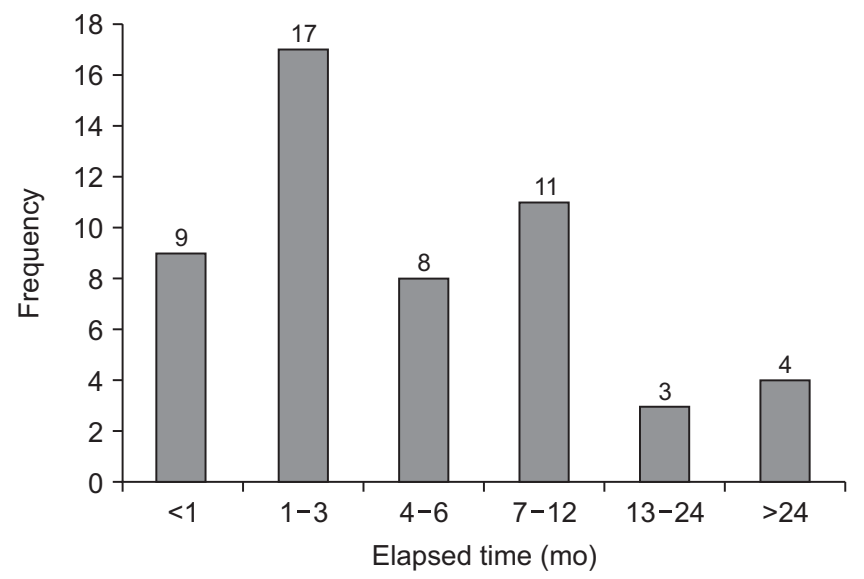

Fig. 2. Elapsed time since the onset of sensory dysfunction.

five subjects with pathologic lesions (9.6\%; Table 2).

Elapsed time from the onset of symptoms to the first clinical visit varied from less than one month to more than two years. Half of the subjects visited the clinic within three months after the onset of symptoms, and $86.5 \%$ of the subjects visited within one year after the onset (Fig. 2).

Hypoesthesia was the most frequent sensory response to tactile stimuli (60.3\%), followed by anesthesia and hyperesthesia. However, the association between the specific type of nerve branch and the response to tactile stimuli was not statistically significant ( $\mathrm{p}=0.637$; Table 3 ). Among the four responses to pin-prick stimuli, analgesia accounted for $36.8 \%$, followed by hyperalgesia $32.4 \%$, and hypoalgesia $27.9 \%$. There was no statistically significant association between the types of nerve branches and the response to pinprick stimuli ( $p=0.260$; Table 4).
There were statistically significant associations between the types of sensory response to the two kinds of stimuli $(\mathrm{p}<0.001)$. Forty cases among $68(58.8 \%)$ showed negative responses to tactile stimulation and pin-prick stimuli (hypoalgesia or analgesia) (Table 5).

\section{DISCUSSION}

The inferior alveolar nerve was the most frequently affected in the study subjects, and tooth extraction and implant surgery were the main causes of sensory dysfunction. These results are in consistent with other studies [18-20]. For example, in a study of 373 patients with post-traumatic trigeminal neuropathy, Van der Cruyssen et al. [18] reported that the inferior alveolar nerve was injured in $45.2 \%$ of patients, maxillary nerve was involved in 34.6\%, and lingual nerve in 14.4\%. According to Klazen et al. [19], among 53 iatrogenic cases of trigeminal nerve injury, the inferior alveolar nerve was the most common with 28 cases, followed by the lingual nerve with 21 cases. Removal of the third molar (24 cases) was the most common iatrogenic trigeminal nerve injury in 53 cases, followed by implant surgery (9 cases) and local anesthesia (9 cases). Choi et al. [20] reported that in 59 patients with sensory impairment after mandibular nerve injury, the most frequent nerve injury involved the inferior alveolar nerve (81\%), and implant surgery (58\%) was the leading cause of nerve injury.

The tactile and pin-prick stimulation of QualST used in this study was intended to mechanically stimulate 
Table 3. Frequency of sensory responses to tactile stimuli

\begin{tabular}{|c|c|c|c|c|c|}
\hline & Normal & Hyperesthesia & Hypoesthesia & Anesthesia & Total \\
\hline \multicolumn{6}{|l|}{ Maxillary nerve } \\
\hline Infraorbital branch & & 2 & 5 & 1 & 8 \\
\hline \multicolumn{6}{|l|}{ Mandibular nerve } \\
\hline Buccal branch & & & 1 & 2 & 3 \\
\hline Inferior alveolar branch & 1 & 8 & 29 & 8 & 46 \\
\hline Lingual branch & 1 & 2 & 6 & 2 & 11 \\
\hline Total & $2(2.9)$ & $12(17.6)$ & $41(60.3)$ & $13(19.1)$ & $68(100.0)$ \\
\hline
\end{tabular}

Values are presented as number (\%). Number of subjects=52 (females, 27; males, 25; mean age 54.0 \pm 16.0 ). In 16 of 52 subjects, the sensory dysfunction involved two independent branches of the trigeminal nerve on the same side. The ratio was calculated as the frequency of specific sensory responses relative to the number of all affected nerve branches. Fisher's exact test, $p=0.637$.

Table 4. Frequency of sensory responses to pin-prick stimuli

\begin{tabular}{|c|c|c|c|c|c|}
\hline & Normal & Hyperalgesia & Hypoalgesia & Analgesia & Total \\
\hline \multicolumn{6}{|l|}{ Maxillary nerve } \\
\hline Infraorbital branch & & 1 & 5 & 2 & 8 \\
\hline \multicolumn{6}{|l|}{ Mandibular nerve } \\
\hline Buccal branch & & & 1 & 2 & 3 \\
\hline Inferior alveolar branch & 2 & 19 & 9 & 16 & 46 \\
\hline Lingual branch & & 2 & 4 & 5 & 11 \\
\hline Total & $2(2.9)$ & $22(32.4)$ & $19(27.9)$ & $25(36.8)$ & $68(100.0)$ \\
\hline
\end{tabular}

Values are presented as number (\%). Number of subjects=52 (females, 27; males, 25; mean age $54.0 \pm 16.0$ ). In 16 of 52 subjects, sensory dysfunction occurred in two independent branches of the trigeminal nerve on the same side. The ratio was calculated as the frequency of specific sensory responses relative to the number of all affected nerve branches. Fisher's exact test, $p=0.260$.

Table 5. Association between sensory responses to tactile and pin-prick stimuli

\begin{tabular}{|c|c|c|c|c|c|}
\hline & Normal & Hyperalgesia & Hypoalgesia & Analgesia & Total \\
\hline Normal & & 2 & & & $2(2.9)$ \\
\hline Hyperesthesia & & 8 & 4 & & $12(17.6)$ \\
\hline Hypoesthesia & 2 & 12 & 13 & 14 & $41(60.3)$ \\
\hline Anesthesia & & & 2 & 11 & $13(19.1)$ \\
\hline Total & $2(2.9)$ & $22(32.4)$ & $19(27.9)$ & $25(36.8)$ & $68(100.0)$ \\
\hline
\end{tabular}

Values are presented as number (\%). Number of subjects=52 (females, 27; males, 25; mean age $54.0 \pm 16.0$ ). In 16 of 52 subjects, sensory dysfunction was reported in two independent branches of the trigeminal nerve on the same side. The ratio was calculated as the frequency of specific sensory responses relative to the number of all affected nerve branches. Fisher's exact test, $p<0.001$.

cutaneous and mucosal sensory receptors of the oral and facial regions. Tactile stimulation via light touch using a cotton swab was assumed to stimulate Merkel's discs, Meissner's corpuscles, and Pacinian corpuscles. Meissner's corpuscles and Merkel's discs, which are innervated by myelinated $A \beta$ nerve fibers, play an essential role in localizing touch sensations. Pacinian corpuscles, which are also innervated by myelinated $A \beta$ nerve fibers, detect vibration or other rapid changes in the tissues [21]. Free nerve endings, primarily afferent $\mathrm{A} \delta$ and $\mathrm{C}$ fibers, detect noxious stimuli. Pin-prick stimulation by the dental explorer evoked an immediate, sharp, and highly localized pain called first pain, which is transmitted through myelinated A $\delta$ fibers [22].

In the present study, most subjects showed hypoesthesia to tactile stimuli, and most subjects showed altered sensory responses to pin-prick stimuli. There was a significant association between the two kinds of stimuli in the patients' responses. These findings are roughly consistent with the results of other studies [18,23]. In a study of 367 patients with post-traumatic trigeminal neuropathy, Van der Cruyssen et al. [18] found that $39.5 \%$ of patients experienced sensory loss. Mechanical hyperesthesia was noted in 15\% of subjects and thermal hyperesthesia reported in 3.8\% of patients with in post-traumatic trigeminal neuropathy. Meewis et 
al. [23] investigated the association between objective and subjective assessments of sensory function in patients diagnosed with post-traumatic trigeminal neuropathy. They found significant associations between objective measurements in most cases.

In this study, some subjects showed a positive response such as hyperesthesia or hyperalgesia to QualST stimuli. Similar results have been reported in other studies [18,23]. Syriatowicz et al. [24] reported that inflammatory mediators such as prostaglandins play a role in the peripheral mechanisms related to hyperalgesia following nerve injury. Hulse [25] proposed a mechanism to enhance sensory neuronal excitability after nerve injury.

Baad-Hansen et al. [17] demonstrated that intra- and inter-examiner reliability of intraoral QualST ranged from 0.63 to 0.75 , which suggested good reliability. Agbaje et al. [26] investigated the agreement between QST and QualST in evaluating orofacial sensory function. Healthy participants were classified as normal according to QST, but as hypersensitive or hyposensitive according to QualST after capsaicin application. Additionally, patients with atypical odontalgia deemed normal using QST were misclassified as hypersensitive, and a small number of patients were classified as hyposensitive by QualST. Ziccardi et al. [27,28] compared clinical neurosensory test results with current perceptual thresholds. Although these two tests yielded similar results for lingual nerve injury, further differences were observed during the assessment of inferior alveolar nerve injury.

This study has several limitations. First, although the number of samples was not small, a larger number would have been desirable to represent the population better. Second, QualST was performed using only two types of stimuli. QualST with diverse stimuli may have resulted in comprehensive evaluation of sensory dysfunction. Third, repeated measurement of the test reliability may have further enhanced the significance of the study results. Fourth, patients' subjective symptoms, which were also important for the evaluation of sensory dysfunction, were not analyzed together with QualST results.

In conclusion, sensory dysfunction can be assessed briefly using simple QualST such as tactile and pin-prick stimulation. QualST represents a convenient screening method for patients with altered orofacial sensation.

\section{CONFLICT OF INTEREST}

No potential conflict of interest relevant to this article was reported.

\section{ACKNOWLEDGEMENTS}

We thank Jisoo Kim in the Department of Education \& Research, Chonnam National University Dental Hospital for processing the medical record data.

\section{ORCID}

\author{
Yeong-Gwan Im \\ https://orcid.org/0000-0003-2703-1475 \\ Byung-Gook Kim \\ https://orcid.org/0000-0002-3602-4720 \\ Jae-Hyung Kim \\ https://orcid.org/0000-0001-5646-4371
}

\section{REFERENCES}

1. Alvarez FK, de Siqueira SR, Okada M, Teixeira MJ, de Siqueira JT. Evaluation of the sensation in patients with trigeminal postherpetic neuralgia. J Oral Pathol Med 2007;36:347-350.

2. Azhary H, Farooq MU, Bhanushali M, Majid A, Kassab MY. Peripheral neuropathy: differential diagnosis and management. Am Fam Physician 2010;81:887-892.

3. Hillerup S. Iatrogenic injury to oral branches of the trigeminal nerve: records of 449 cases. Clin Oral Investig 2007;11:133-142.

4. Kim YK, Kim SG, Kim JH. Altered sensation after orthognathic surgery. J Oral Maxillofac Surg 2011;69:893-898.

5. Petersen LØ, Ipsen EØ, Felding UA, von Buchwald C, Steinmetz J. Sequelae of major trauma patients with maxillofacial fractures. Ann Otol Rhinol Laryngol 2021;130:475-482.

6. Silva NSV, da Silva LA, Jaluul 0, Jacob-Filho W, Siqueira SRDT. Oral infections, comorbidities and sensory evidences in elderly: cross-sectional study. Arch Gerontol Geriatr 2017;73:15-20.

7. Tay $A B$, Lai JB, Lye KW, et al. Inferior alveolar nerve injury in trauma-induced mandible fractures. J Oral Maxillofac Surg 2015;73:1328-1340.

8. Essick GK, Phillips C, Turvey TA, Tucker M. Facial altered sensation and sensory impairment after orthognathic surgery. Int J Oral Maxillofac Surg 2007;36:577-582.

9. Svensson P, Baad-Hansen L, Pigg M, et al.; Special Interest Group of Oro-facial Pain. Guidelines and recommendations for assessment of somatosensory function in oro-facial pain conditions--a taskforce report. J Oral Rehabil 2011;38:366-394.

10. Van der Cruyssen F, Van Tieghem L, Croonenborghs TM, et al. 
Orofacial quantitative sensory testing: current evidence and future perspectives. Eur J Pain 2020;24:1425-1439.

11. Kim YK, Yun PY, Kim JH, Lee JY, Lee W. The quantitative sensory testing is an efficient objective method for assessment of nerve injury. Maxillofac Plast Reconstr Surg 2015;37:13.

12. Rolke R, Baron R, Maier C, et al. Quantitative sensory testing in the German Research Network on Neuropathic Pain (DFNS): standardized protocol and reference values. Pain 2006;123:231-243.

13. Rolke R, Magerl W, Campbell KA, et al. Quantitative sensory testing: a comprehensive protocol for clinical trials. Eur J Pain 2006;10:77-88.

14. Jääskeläinen SK. The utility of clinical neurophysiological and quantitative sensory testing for trigeminal neuropathy. J Orofac Pain 2004;18:355-359.

15. Yekta SS, Koch F, Grosjean MB, et al. Analysis of trigeminal nerve disorders after oral and maxillofacial intervention. Head Face Med 2010;6:24.

16. Jacobs R, Wu CH, Goossens K, Van Loven K, Van Hees J, Van Steenberghe D. Oral mucosal versus cutaneous sensory testing: a review of the literature. J Oral Rehabil 2002;29:923-950.

17. Baad-Hansen L, Pigg M, Ivanovic SE, et al. Chairside intraoral qualitative somatosensory testing: reliability and comparison between patients with atypical odontalgia and healthy controls. J Orofac Pain 2013;27:165-170.

18. Van der Cruyssen F, Peeters F, De Laat A, Jacobs R, Politis C, Renton T. Prognostic factors, symptom evolution, and quality of life of posttraumatic trigeminal neuropathy. Pain 2021. doi: 10.1097/j.pain.0000000000002408. [Epub ahead of print]

19. Klazen Y, Van der Cruyssen F, Vranckx M, et al. Iatrogenic trigeminal post-traumatic neuropathy: a retrospective two-year cohort study. Int J Oral Maxillofac Surg 2018;47:789-793.

20. Choi YC, Kwon JS, Kim ST, Ahn HJ. Analysis of patients with dysesthesia after mandibular nerve injury. J Oral Med Pain 2009;34:379-385.

21. Hall JE, Hall ME. Guyton and Hall textbook of medical physiology. 14th ed. Philadelphia: Elsevier; 2021. pp. 599-600.

22. Feher J. Quantitative human physiology: an introduction. San Diego: Academic Press; 2012. pp. 321-331.

23. Meewis J, Renton T, Jacobs R, Politis C, Van der Cruyssen F. Posttraumatic trigeminal neuropathy: correlation between objective and subjective assessments and a prediction model for neurosensory recovery. J Headache Pain 2021;22:44.

24. Syriatowicz JP, Hu D, Walker JS, Tracey DJ. Hyperalgesia due to nerve injury: role of prostaglandins. Neuroscience 1999;94:587594.

25. Hulse RP. Identification of mechano-sensitive $C$ fibre sensitization and contribution to nerve injury-induced mechanical hyperalgesia. Eur J Pain 2016;20:615-625.

26. Agbaje J, De Laat A, Constantinus P, Svensson P, Baad-Hansen L. Agreement between quantitative and qualitative sensory testing of changes in oro-facial somatosensory sensitivity. J Oral Rehabil 2017;44:30-42.

27. Ziccardi VB, Hullett JS, Gomes J. Physical neurosensory testing versus current perception threshold assessment in trigeminal nerve injuries related to dental treatment: a retrospective study. Quintessence Int 2009;40:603-609.

28. Ziccardi VB, Dragoo J, Eliav E, Benoliel R. Comparison of current perception threshold electrical testing to clinical sensory testing for lingual nerve injuries. J Oral Maxillofac Surg 2012;70:289294. 